\title{
A glimpse at the flat-spacetime limit of quantum gravity using the Bekenstein argument in reverse ${ }^{1}$
}

\author{
Giovanni AMELINO-CAMELIA ${ }^{a}$, Michele ARZANO $^{b}$, Andrea PROCACCINI $^{a}$ \\ ${ }^{a}$ Dip. Fisica Univ. Roma "La Sapienza" and Sez. Roma1 INFN, Piazzale Moro 2, Roma, Italy \\ ${ }^{b}$ Dept Physics and Astronomy, University of North Carolina, Chapel Hill, NC 27599, USA
}

\begin{abstract}
An insightful argument for a linear relation between the entropy and the area of a black hole was given by Bekenstein using only the energy-momentum dispersion relation, the uncertainty principle, and some properties of classical black holes. Recent analyses within String Theory and Loop Quantum Gravity describe black-hole entropy in terms of a dominant contribution, which indeed depends linearly on the area, and a leading log-area correction. We argue that, by reversing the Bekenstein argument, the log-area correction can provide insight on the energy-momentum dispersion relation and the uncertainty principle of a quantum-gravity theory. As examples we consider the energy-momentum dispersion relations that recently emerged in the Loop Quantum Gravity literature and the Generalized Uncertainty Principle that is expected to hold in String Theory.
\end{abstract}

As first observed by Bekenstein [1], the fact that the entropy of a black hole should be proportional to its (horizon-surface) area, up to corrections that can be neglected when the area $A$ is much larger than the square of the Planck length $L_{p}$, can be derived using very simple ingredients. In (classical) general-relativity one finds [2] that the minimum increase of area when the black hole absorbs a classical particle of energy $E$ and size $s$ is $\Delta A \simeq 8 \pi L_{p}^{2} E s$ (in "natural units" with $\hbar=c=1$ ). Taking into account the quantum properties of particles one can estimate the size of the particle as roughly given by its position uncertainty $\delta x$, and one can assume that a particle with position uncertainty $\delta x$ should at least [3] have energy $E \sim 1 / \delta x$. This leads to the conclusion[1, 4] that the minimum change in the black-hole area must be of order $L_{p}^{2}$, independently of the size of the area. Then using the fact that, also independently of the size of the area, this minimum increase of area should correspond to the minimum ("one bit") change of entropy, $(\Delta S)_{\min }=\ln 2$, one easily obtains [1] the proportionality between black-hole entropy and area.

Over the last few years both in String Theory and in Loop Quantum Gravity some techniques for the analysis of entropy on the basis of quantum properties of black holes were developed. These results $[5,6]$ now go even beyond the entropy-area-proportionality contribution: they establish that

\footnotetext{
${ }^{1}$ This essay received an "honorable mention" in the 2004 Essay Competition of the Gravity Research Foundation Ed.
} 
the leading correction should be of log-area type, so that one expects (for $A \gg L_{p}^{2}$ ) an entropy-area relation for black holes of the type

$$
S=\frac{A}{4 L_{p}^{2}}+\rho \ln \frac{A}{L_{p}^{2}}+O\left(\frac{L_{p}^{2}}{A}\right) .
$$

While all analyses agree on the coefficient of the term $A / L_{p}^{2}$, different quantum-gravity theories appear to give rise $[5,6,7]$ to different predictions for the coefficient $\rho$ of the leading $\ln \left(A / L_{p}^{2}\right)$ correction.

Here we propose that the log-area leading correction might admit a simple description, just like the argument presented by Bekenstein in Ref. [1] gives a simple description of the dominant linearin- $A$ contribution. Our simple description will also explain why the coefficient $\rho$ of the log-area term takes different values in different quantum-gravity theories. And we stress that the availability of results on the log-area correction might provide motivation for reversing the Bekenstein argument: the knowledge of the black-hole entropy-area law up to the leading log correction can be used to establish the Planck-scale modifications of the ingredients of the Bekenstein analysis.

We start by observing that, as stressed above, a key point for the Bekenstein analysis is the $E \geq 1 / \delta x$ relation between the energy of a particle and the uncertainty in its position. One obtains $E \geq 1 / \delta x$ by combining appropriately [3] the Heisenberg uncertainty principle, the special-relativistic energy-momentum dispersion relation, and the fact that a procedure for the measurement of the position of a particle must not introduce an energy uncertainty for the particle which is greater than its mass (in its rest frame), since otherwise the procedure is subject to the possibility of production of additional copies of the particle. But nearly all approaches to the quantum-gravity problem predict a Planck-length-dependent modification of $E \geq 1 / \delta x$, since various quantum-gravity results suggest that the uncertainty principle and/or the energy-momentum dispersion relation should be modified. Modifications of the uncertainty principle have been discussed in quantum-gravity theories that predict a new role for the Planck length in the localization of particles, as in the case of String Theory [8]. Several scenarios for a modified energy-momentum dispersion relation [9] have been considered, particularly in quantum pictures of spacetime which involve some level of spacetime discreteness [10] or noncommutativity $[11,12,13,14]$. In Loop Quantum Gravity, which indeed predicts a certain form of spacetime granularity (discrete spectrum of areas and volumes), several studies[15, 16] found evidence for a modification of the dispersion relation.

In order to accommodate the differences between alternative quantum-gravity scenarios we consider a rather general parametrization of the Planck-length modified $E \geq 1 / \delta x$ relation:

$$
E \geq \frac{1}{\delta x}-\eta_{1} \frac{L_{p}}{(\delta x)^{2}}-\eta_{2} \frac{L_{p}^{2}}{(\delta x)^{3}}+O\left(\frac{L_{p}^{3}}{(\delta x)^{4}}\right)
$$

We will argue that a log-area leading correction to the entropy-area relation requires $\eta_{1}=0$. For $\eta_{1} \neq 0$ the leading correction turns out to behave like the square-root of the area. A result in agreement with 
(1) is obtained for $\eta_{1}=0, \eta_{2} \sim \rho$ (and the sign convention in (2) turns out to ensure that $\eta_{2}$ and $\rho$ are either both positive or both negative).

As in the original Bekenstein argument [1], we take as starting point the general-relativity result which establishes that the area of a black hole changes according to $\Delta A \geq 8 \pi E s$ when a classical particle of energy $E$ and size $s$ is absorbed. In order to describe the absorption of a quantum particle one must describe the size of the particle in terms of the uncertainty in its position [1,4], $s \sim \delta x$, and take into account a "calibration factor" $[17,18,19](\ln 2) / 2 \pi$ that connects the $\Delta A \geq 8 \pi E s$ classical-particle result with the quantum-particle estimate $\Delta A \geq 4(\ln 2) L_{p}^{2} E \delta x$. Following the original Bekenstein argument [1] one then enforces the relation $E \geq 1 / \delta x\left(\right.$ and this leads to $\left.\Delta A \geq 4(\ln 2) L_{p}^{2}\right)$, but we must take into account the Planck-length modification in (2), obtaining

$$
\Delta A \geq 4(\ln 2)\left[L_{p}^{2}-\frac{\eta_{1} L_{p}^{3}}{\delta x}-\frac{\eta_{2} L_{p}^{4}}{(\delta x)^{2}}\right] \simeq 4(\ln 2)\left[L_{p}^{2}-\frac{\eta_{1} L_{p}^{3}}{R_{S}}-\frac{\eta_{2} L_{p}^{4}}{\left(R_{S}\right)^{2}}\right] \simeq 4(\ln 2)\left[L_{p}^{2}-\frac{\eta_{1} 2 \sqrt{\pi} L_{p}^{3}}{\sqrt{A}}-\frac{\eta_{2} 4 \pi L_{p}^{4}}{A}\right]
$$

where we also used the fact that in falling in the black hole the particle acquires $[18,21,22]$ position uncertainty $\delta x \sim R_{S}$, where $R_{S}$ is the Schwarzschild radius (and of course $A=4 \pi R_{S}^{2}$ ).

Next, following again Bekenstein[1], one assumes that the entropy depends only on the area of the black hole, and one uses the fact that according to information theory the minimum increase of entropy should be $\ln 2$, independently of the value of the area:

$$
\frac{d S}{d A} \simeq \frac{\min (\Delta S)}{\min (\Delta A)} \simeq \frac{\ln 2}{4(\ln 2) L_{p}^{2}\left[1-\eta_{1} 2 \sqrt{\pi} \frac{L_{p}}{\sqrt{A}}-\eta_{2} 4 \pi \frac{L_{p}^{2}}{A}\right]} \simeq\left(\frac{1}{4 L_{p}^{2}}+\frac{\eta_{1} \sqrt{\pi}}{2 L_{p} \sqrt{A}}+\frac{\eta_{2} \pi}{A}\right) .
$$

From this one easily obtains (up to an irrelevant constant contribution to entropy):

$$
S \simeq \frac{A}{4 L_{p}^{2}}+\eta_{1} \sqrt{\pi} \frac{\sqrt{A}}{L_{p}}+\eta_{2} \pi \ln \frac{A}{L_{p}^{2}} .
$$

As anticipated the sought agreement with (1) requires $\eta_{1}=0$ and $\eta_{2} \sim \rho$ (precisely $\eta_{2}=\rho / \pi$ ).

From this we conjecture that the log-area contribution to the black-hole entropy might be deeply connected with a corresponding $L_{p}^{2} /(\delta x)^{3}$ correction to the $E \geq 1 / \delta x$ relation. And, as anticipated, our description encourages the possibility that the coefficient of the log-area correction might be different in different quantum-gravity theories. The dominant linear-in- $A$ term must be "universal" since it was obtained in Ref. [1] using only observations that do not involve any quantum-gravity effects (the Planck length appears in the Bekenstein formula only as a result of the classical-gravity aspects of the analysis, which of course involve the gravitational constant). Instead the coefficient of the log-area leading correction depends directly on the coefficient of the $L_{p}^{2} /(\delta x)^{3}$ correction to the $E \geq 1 / \delta x$ relation, and, since different quantum-gravity theories could lead to different modifications of the Heisenberg uncertainty principle and of the energy-momentum dispersion relation, this coefficient should not be expected to take the same value in all quantum-gravity theories. 
Amusingly this observation provides an opportunity to use the Bekenstein argument in reverse. In the 1970s there was no result on entropy from the analysis of quantum properties of black holes and analyses "a la Bekenstein", using some simple ingredients, allowed to derive the linear term in the entropy-area formula. Now that the analysis of quantum properties of black holes allows to derive also the log-area correction we might be able to use this information to infer which Planck-scale modifications should be introduced in the ingredients of the Bekenstein analysis.

A first application of this "reversed Bekenstein argument" can be found in Loop Quantum Gravity. There is a large number of Loop-Quantum-Gravity studies (see, e.g., Refs. [15, 16] and references therein) reporting results in support of the possibility of a Planck-scale modified energy-momentum dispersion relation, although there is still no consensus on the precise form of this modification. The ideal framework for establishing Planck-scale modifications of the dispersion relation is the study of the self-energy of a particle in a low-energy effective theory on "quasi-Minkowski spacetime" (a quantum spacetime which most closely approximates Minkowski spacetime in a given quantum-gravity theory). But in Loop Quantum Gravity the well-known "classical-limit problem" [23], which of course includes a "Minkowski-limit problem", has provided so far a rather serious obstacle for the analysis of Planckscale modifications of dispersion relations. In particular, two key alternatives for the Planck-scale modification of the energy-momentum dispersion relation are still being considered [15, 16]: one in which the function $p(E)$ admits an expansion with leading Planck-scale correction of order $L_{p} E^{3}$,

$$
\vec{p}^{2} \simeq E^{2}-m^{2}+\alpha_{1} L_{p} E^{3}
$$

and one in which the function $p(E)$ admits an expansion with leading Planck-scale correction of order $L_{p}^{2} E^{4}$,

$$
\vec{p}^{2} \simeq E^{2}-m^{2}+\alpha_{2} L_{p}^{2} E^{4} .
$$

We intend to show that, since also in Loop Quantum Gravity there is evidence [6] that the leading correction to the entropy-area black-hole formula is logarithmic, our "reversed Bekenstein argument" favors the scenario (6). The scenario (5) would lead to a $\sqrt{A}$ leading correction.

On the basis of the analysis reported above it will be sufficient to study the implications of these modified dispersion relations for the relation between the energy of a particle and its position uncertainty, and show that (6) leads to a contribution of the type $L_{p}^{2} /(\delta x)^{3}$, while from (5) one obtains a $L_{p} /(\delta x)^{2}$ term.

In order to establish the implications for the relation between the energy of a particle and its position uncertainty, we can follow the familiar derivation [3] of the relation $E \geq 1 / \delta x$, substituting, where applicable, the standard special-relativistic dispersion relation with the Planck-scale modified dispersion relation. Let us first consider the case of the dispersion relation (6). It is convenient to start by focusing on the case of a particle of mass $M$ at rest, whose position is being measured by a procedure involving a collision with a photon of energy $E_{\gamma}$ and momentum $p_{\gamma}$. In order to measure the 
particle position with precision $\delta x$ one should use a photon with momentum uncertainty $\delta p_{\gamma} \geq 1 / \delta x$. This $\delta p_{\gamma} \geq 1 / \delta x$ relation originates from Heisenberg's uncertainty principle for which, as mentioned, modifications have not been suggested in the Loop-Quantum-Gravity literature (but they are expected in other approaches to the quantum-gravity problem). Following the standard argument [3], one takes this $\delta p_{\gamma} \geq 1 / \delta x$ relation and converts it into the relation $\delta E_{\gamma} \geq 1 / \delta x$, using the special-relativistic dispersion relation, and then the relation $\delta E_{\gamma} \geq 1 / \delta x$ is converted into the relation $M \geq 1 / \delta x$ because the measurement procedure requires ${ }^{2} M \geq \delta E_{\gamma}$. If indeed Loop Quantum Gravity hosts a Planckscale-modified dispersion relation of the form (6), it is easy to see that, following the same reasoning, one would obtain from $\delta p_{\gamma} \geq 1 / \delta x$ the requirement

$$
M \geq \frac{1}{\delta x}\left(1-\alpha_{2} \frac{3 L_{p}^{2}}{2(\delta x)^{2}}\right) .
$$

These results strictly apply only to the measurement of the position of a particle at rest, but they can be straightforwardly generalized [3] (simply using a boost) to the case of measurement of the position of a particle of energy $E$. In the case of the standard dispersion relation (without Planckscale modification) one obtains the familiar $E \geq 1 / \delta x$, as required for a purely linear dependence of entropy on area. In the case of (6) one instead easily finds that $E \geq \frac{1}{\delta x}\left(1-\alpha_{2} \frac{3 L_{p}^{2}}{2(\delta x)^{2}}\right)$, which fulfills the requirements of our derivation of a leading-order correction of log-area form.

The careful reader can easily adapt to the case of the dispersion relation (5) this simple analysis which we discussed for the dispersion relation (6). The end result is that from (5) it follows that $E \geq \frac{1}{\delta x}\left(1+\alpha_{1} \frac{L_{p}}{\delta x}\right)$, and this in turn leads to a leading correction to the entropy-area formula that goes like the square-root of the area, rather than the predicted log-area leading correction. This confirms that, using the reversed Bekenstein argument, the dispersion relation (6) should be preferred to the dispersion relation (5).

In closing let us turn to String Theory. Also in String Theory there are robust indications of a logarithmic contribution to the black-hole entropy-area relation, but at present the mainstream StringTheory literature provides no evidence for a Planck-scale modification of the dispersion relation. In the case of String Theory our proposed "reversed Bekenstein argument" leads us to consider the so-called "GUP" or "Generalized Uncertainty Principle",

$$
\delta x \geq \frac{1}{\delta p}+\lambda_{s}^{2} \delta p
$$

which finds support in various String-Theory studies [8]. The scale $\lambda_{s}$ in (8) is an effective string length (a characteristic length scale of the GUP which should be closely related to the string length and therefore the Planck length).

The presence of a logarithmic term in the entropy-area relation can be viewed as a consequence of the presence of the $\lambda_{s}^{2} \delta p$ term in the GUP. In fact, as the careful reader can easily verify, from the

\footnotetext{
${ }^{2}$ We are here again using the fact [3] that the measurement procedure must ensure that the relevant energy uncertainties are not large enough to possibly produce extra copies of the particle whose position one intends to measure.
} 
GUP one obtains (following again straightforwardly the standard measurability line of analysis [3]) a modification of the relation $E \geq 1 / \delta x$. The modification is of the type $E \geq 1 / \delta x+\Delta$, with $\Delta$ of order $\lambda_{s}^{2} / \delta x^{3}$, and originates from the fact that according to the GUP, (8), one obtains $\delta p_{\gamma} \geq 1 / \delta x+\lambda_{s}^{2} / \delta x^{3}$, instead of the $\delta p_{\gamma} \geq 1 / \delta x$ which follows from Heinsenberg's uncertainty principle. As discussed above this type of relation between the energy and the position uncertainty of a particle, with a $1 / \delta x^{3}$ contribution in addition to the familiar $1 / \delta x$ term, leads, through the Bekenstein argument, to a logarithmic contribution to the black-hole entropy-area relation.

The examples of Loop Quantum Gravity and String Theory show that we might have at our disposal a rather powerful tool for the analysis of quantum-gravity theories, assuming that it is legitimate to think of a connection between the log-area contribution to black-hole entropy and a $1 /(\delta x)^{3}$ correction to the $E \geq 1 / \delta x$ relation. Whereas in Loop Quantum Gravity the $L_{p}^{2} /(\delta x)^{3}$ correction is motivated by results on Planck-scale-modified dispersion relations of the type (6), in String Theory the analogous correction of order $\lambda_{s}^{2} / \delta x^{3}$ is motivated by the GUP. In general, in any given quantum-gravity theory, the presence of a logarithmic term in the black-hole entropy-area relation may be a manifestation of a modified dispersion relation and/or a modified uncertainty principle.

\section{Acknowledgments}

G. A.-C. gratefully acknowledges conversations with O. Dryer, D. Oriti, C. Rovelli and L. Smolin. The work of M. A. was supported by a Fellowship from The Graduate School of The University of North Carolina. M. A. also thanks the Department of Physics of the University of Rome for hospitality.

\section{References}

[1] J. D. Bekenstein, Phys. Rev. D7 (1973) 2333.

[2] D. Christodoulou, Phys. Rev. Lett. 25 (1970) 1596; D. Christodoulou and R. Ruffini, Phys. Rev. D4 (1971) 3552 .

[3] E. M. Lifshitz, L. P. Pitaevskii and V. B. Berestetskii, "Landau-Lifshitz Course of Theoretical Physics, Volume 4: Quantum Electrodynamics" (Reed Educational and Professional Publishing, 1982).

[4] S. Hod, Phys. Rev. Lett. 81 (1998) 4293.

[5] A. Strominger and C. Vafa, Phys. Lett. B379 (1996) 99; S. N. Solodukhin, Phys. Rev. D57 (1998) 2410.

[6] C. Rovelli, Phys. Rev. Lett. 77 (1996) 3288; A. Ashtekar, Phys. Rev. Lett. 80 (1998) 904; R.K. Kaul and P. Majumdar, Phys. Rev. Lett. 84 (2000) 5255.

[7] S. Das, P. Majumdar and R. K. Bhaduri, hep-th/0111001, Class. Quant. Grav. 19 (2002) 2355; A. Ghosh and P. Mitra, gr-qc/0401070; G. Gour and A.J.M. Medved, Class. Quant. Grav. 20 (2003) 3307; S. Das, hep-th/0403202.

[8] G. Veneziano, Europhys. Lett. 2 (1986) 199; D.J. Gross and P.F. Mende, Nucl. Phys. B303 (1988) 407; D. Amati, M. Ciafaloni and G. Veneziano, Phys. Lett. B216 (1989) 41. 
[9] G. Amelino-Camelia, J. Ellis, N.E. Mavromatos and D.V. Nanopoulos, hep-th/9605211, Int. J. Mod. Phys. A12 (1997) 607; G. Amelino-Camelia, J. Ellis, N.E. Mavromatos, D.V. Nanopoulos and S. Sarkar, astro-ph/9712103, Nature 393 (1998) 763.

[10] G. 't Hooft, Class. Quant. Grav. 13 (1996) 1023.

[11] S. Majid and H. Ruegg, Phys. Lett. B334 (1994) 348.

[12] J. Lukierski, H. Ruegg and W.J. Zakrzewski Ann. Phys. 243 (1995) 90.

[13] G. Amelino-Camelia, gr-qc/0012051, Int. J. Mod. Phys. D11 (2002) 35; Jerzy Kowalski-Glikman and S. Nowak, hep-th/0204245, Int. J. Mod. Phys. D12 (2003) 299; G. Amelino-Camelia, gr-qc/0207049, Nature 418 (2002) 34 .

[14] A. Agostini, G. Amelino-Camelia and F. D'Andrea, hep-th/0306013.

[15] R. Gambini and J. Pullin, Phys. Rev. D59 (1999) 124021; J. Alfaro, H.A. Morales-Tecotl and L.F. Urrutia, Phys. Rev. Lett. 84 (2000) 2318.

[16] G. Amelino-Camelia, L. Smolin and A. Starodubtsev, hep-th/0306134.

[17] P. Chen and R.J. Adler, Nucl. Phys. Proc. Suppl. 124 (2003) 103.

[18] P. S. Custodio and J.E. Horvath, Class. Quant. Grav. 20 (2003) L197.

[19] T. Damour, hep-th/0401160; M. Cavaglia, S. Das and R. Maartens, Class. Quant. Grav. 20 (2003) L205.

[20] S.W. Hawking, Nature 248 (1974) 30.

[21] M.Yu. Kuchiev and V.V. Flambaum, gr-qc/0312065.

[22] R.J. Adler and T.K. Das, Phys. Rev. D14 (1976) 2472; R.S. Hanni and R. Ruffini, "Lines of Force of a point Charge Near a Schwarzschild Black Hole", in Black Holes, eds. C. DeWitt and B.S. DeWitt (Gordon Breach 1973).

[23] L. Smolin, hep-th/0303185. 